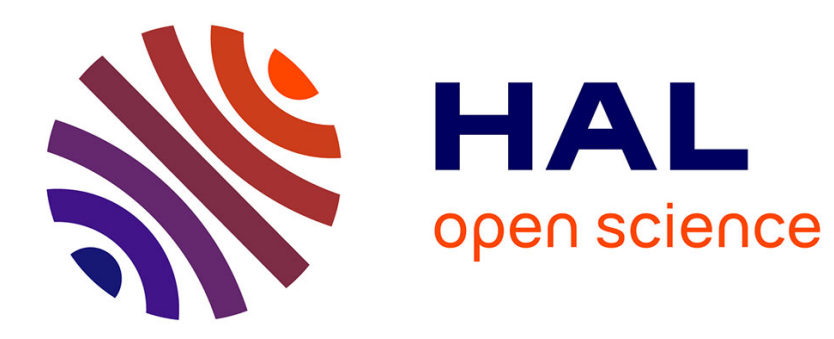

\title{
De la violence ordinaire dans les organisations
}

Pascal Fugier

\section{To cite this version:}

| Pascal Fugier. De la violence ordinaire dans les organisations. 2014. hal-01682105

\section{HAL Id: hal-01682105 \\ https://hal.science/hal-01682105}

Submitted on 12 Jan 2018

HAL is a multi-disciplinary open access archive for the deposit and dissemination of scientific research documents, whether they are published or not. The documents may come from teaching and research institutions in France or abroad, or from public or private research centers.
L'archive ouverte pluridisciplinaire HAL, est destinée au dépôt et à la diffusion de documents scientifiques de niveau recherche, publiés ou non, émanant des établissements d'enseignement et de recherche français ou étrangers, des laboratoires publics ou privés. 


\section{Pascal Fugier (2014), De la violence ordinaire dans les organisations. Gérer et comprendre, 115(1), 74-76.}

À propos du livre de Gilles Herreros, La violence ordinaire dans les organisations.
Plaidoyer pour des organisations réflexives, Toulouse, Éditions Érès, 2012

Gilles Herreros défend une approche clinico-critique dans l'étude des organisations auprès desquelles il intervient. Ainsi, dans l'ouvrage précité qui retrace neuf situations de violence ordinaire dans des organisations, ce sociologue affirme que sa fonction n'est pas de réguler les situations qui se dressent face à lui, mais de " provoquer une réflexion qui place dans le questionnement et dans l'intranquillité les normes régulatrices » (p. 14) du milieu professionnel qu'il étudie. La posture de l'auteur nous rappelle celle des tenants de l'analyse institutionnelle (tels René Lourau ou Georges Lapassade) identifiant le psychosociologue à un « analyseur » qui fait surgir les non-dits d'une institution en la provoquant.

La sociologie critique de Gilles Herreros s'affiche comme une entreprise d'explicitation et de déconstruction de ce qui est établi dans les organisations. Ceci dit, s'il les interpelle, il ne vise pas pour autant leur disparition ni même leur simple affaiblissement: il s'emploie à instituer en leur sein un cadre dans lequel se déploient des formes de réflexivité qui œuvrent à "l'énonciation de ce qui, au quotidien, use les salariés et les abuse » (p. 14). L'établissement durable d'un tel cadre, sous la forme d'une organisation réflexive, constitue le principal enjeu de cette sociologie d'intervention qui revendique la compatibilité entre la posture clinique et la sociologie critique.

Gilles Herreros définit les principes sur lesquels repose sa posture clinique. Celleci s'incarne dans le refus de prendre la place de l'expert, du maître d'un savoir. S'il délivre ses interprétations et soumet quelques hypothèses à ses interlocuteurs, le travail d'objectivation que mène méthodiquement le sociologue ne vise pas à révéler la vérité objective de l'organisation. Et plutôt que de renoncer à sa propre subjectivité, il inclut l'explicitation de cette dernière dans son procès de recherche. Corrélativement, considérant la double face de sujets à la fois assujettis et résistants à ce qui les détermine, Gilles Herreros reconnaît aux individus leur 
capacité réflexive et leur négativité, c'est-à-dire leur capacité à nier l'existant, à prendre leurs distances avec le réel. Certes, comme dans la sociologie de Robert Castel, leur capacité de résistance est conditionnée à la détention de certains supports sociaux (ressources économiques, relationnelles, culturelles...). Mais la posture clinique défendue par le sociologue valorise et renforce la réflexivité des sujets en instituant « une mise en travail, une mise en crise de ce qui [assujettit] » (p. 52) les individus en situation de travail. Dès lors, la scientificité a pour terrain d'expression l'intersubjectivité, c'est-à-dire "la rencontre et la confrontation des subjectivités » (p. 52), et elle se conçoit comme le produit de la «controverse démocratique» (p. 20) entre le chercheur et ses interlocuteurs.

La thématique de recherche abordée par l'auteur n'a a priori rien d'inédit, ni dans le champ des sciences humaines ni dans le champ politico-médiatique. Depuis le choc provoqué par des suicides chez Renault (Guyancourt) et à France Télécom, plusieurs rapports ministériels consacrés à la prévention des risques psychosociaux se sont particulièrement penchés sur la souffrance et le mal-être au travail. Et, corrélativement, de nombreux chercheurs commentés par l'auteur, comme Christophe Dejours et Vincent de Gaulejac, privilégient cette terminologie. Gilles Herreros tient pour sa part à rendre compte de la violence dans l'organisation en démontrant comment ce mal agir, par son invisibilité et sa banalisation, «engendre du pâtir» (p. 13). Le choix du terme de violence, plutôt que ceux de souffrance et de mal-être, n'est pas anodin, et on en perçoit toute la valeur opératoire au fur et à mesure des situations narrées dans l'ouvrage. Gilles Herreros refuse de ramener l'ordinaire de la souffrance au travail à son seul contexte sociohistorique d'émergence (l'hyper-modernité, la lean production, l'idéologie managériale...). Or, la notion de souffrance a de fait encouragé un raisonnement «exclusivement macrologique» (p. 28), qui ne peut que générer un sentiment de fatalité et diluer les responsabilités (p. 29), de la même manière que l'usage non contrôlé du terme de harcèlement tend à psychologiser à outrance l'expérience de la domination au travail. De plus, l'extension abusive du concept de souffrance à un ensemble de phénomènes disparates (stress, dépression, troubles musculosquelettiques...) atteste du flou sémantique qui entoure cette notion.

En retournant au concept de violence, Gilles Herreros gagne en précision. Il analyse dans le détail la manière dont celle-ci « s'incarne dans des êtres, des comportements, 
des modes de faire» (p. 30). Ce qui le conduit aussi à remettre en question le concept de domination symbolique, une domination qui met certes en évidence l'imposition de significations par un groupe social dominant, mais sans supposer que celui-ci ait conscience d'exercer une telle violence, ce qui a là encore pour conséquence de «désengager la responsabilité des individus et des collectifs qui provoquent le pâtir» (p. 35).

Nous n'allons pas revenir en détail sur chacune des situations d'intervention que Gilles Herreros livre dans son ouvrage, des situations qui touchent aux domaines du soin, de l'éducation, de l'économie sociale et solidaire, de l'université, de la recherche scientifique, de l'ingénierie publique, du travail social et de l'informatique. Nous nous en tiendrons plutôt à ses problématiques transversales.

Gilles Herreros insiste sur le fait que la violence dans les organisations a tendance à se concentrer sur «un coeur de cible» (p. 77), principalement (mais pas exclusivement) parmi les catégories du personnel les moins «légitimes» (magasiniers, secrétaires, techniciennes en formation...), même si elle se propage à l'ensemble de l'organisation et constitue une expérience assez largement partagée (attestant ainsi de son caractère systémique).

Ainsi, dans le récit de son intervention menée au sein d'un hôpital, il repère certes les incidences néfastes sur l'ensemble du personnel de la rationalisation de l'organisation du travail qu'impose la révision générale des politiques publiques (RGPP), mais il remarque également que ce sont surtout les magasiniers, dont le travail est le plus méprisé, qui font en l'occurrence office de boucs émissaires. Ils réagissent à ces formes d'agression par des stratégies collectives de défense (refus d'obtempérer, freinage...) afin d'assurer leur «survie symbolique» (p. 75), ce qui est interprété par le reste du personnel comme la marque de leur résistance au changement et de leur mauvaise volonté. Gilles Herreros met alors en avant la responsabilité de cadres qui plutôt que de relayer et d'accentuer «la boucémissarisation de quelques-uns » auraient tout intérêt «à risquer des analyses et une problématisation de la situation » (p. 78).

Les cadres de direction d'un lycée professionnel sont eux aussi interpellés par le chercheur. Ne constituant pas les relais des enseignants, leur alliance avec 
l'autorité de tutelle ne peut être interprétée que comme une forme de désaveu par le corps enseignant, qui voit en eux « des censeurs, des évaluateurs au service d'une autorité bureaucratique » (p. 91).

L'intervention menée par l'auteur au sein d'une entreprise relevant du champ de l'économie sociale et solidaire lui permet de rendre compte de la violence qu'exercent les cadres lorsqu'ils confondent leur nécessaire autorité avec l'autoritarisme dans le contexte d'une modernisation de l'entreprise marquée par le passage brutal « de l'ère des militants à celle des gestionnaires (p. 97).

La crise symbolique que traverse cette entreprise prend la forme d'une «mise $\grave{a}$ mort symbolique » (p. 118) dans une autre situation présentée par le sociologue, celle du directeur d'un laboratoire de recherche dont le successeur conteste chacune de ses prises de parole et n'hésite pas à l'humilier pour asseoir sa propre «autorité». L'humiliation constitue le trait marquant d'autres situations d'intervention relatées par Gilles Herreros : les interactions entre une conseillère en formation et sa tutrice au sein d'une Chambre de Commerce, le sort réservé à des techniciens et à des ingénieurs relevant du ministère de l'Environnement et du Développement durable, qui subissent un déclassement de leur statut en étant soudainement qualifiés d'« improductifs » faisant office de « fonctions supports » (p. 133).

S'il est difficile pour ces techniciens et ingénieurs de supporter la stigmatisation d'être considérés comme des "has been", la situation que subit Maxime, un ingénieur informaticien, nous rappelle que la violence ordinaire dans les organisations n'est pas que symbolique, mais peut aussi être physique. Menacé par un chef de service qui le somme de respecter son territoire s'il veut " rester en vie ", Maxime se remémore ce jour où l'un de ses collègues cadres a été plaqué contre le mur par un responsable de son département car il avait eu la mauvaise idée d'investir «le territoire » de ce dernier. La passivité dont fait preuve Maxime face à ce type de situation prend sens quand on comprend que les nombreux avantages dont il bénéficie au sein de cette entreprise (nombre important de jours de congés payés, salaire élevé...), constituent une force contraire à son désir d'opposition, une situation paradoxale qu'il résume en disant qu'il est tenu "par une chaîne en or » (p. 156). 
Au-delà de cette mise en perspective originale et productive de la violence ordinaire dans les organisations (tant dans son empiricité que dans sa conceptualité), on appréciera aussi les précisions méthodologiques que dissémine Gilles Herreros au gré de ses différents récits d'intervention. Elles permettent au lecteur de se mettre au plus près du travail d'un sociologue clinicien, notamment s'agissant de l'analyse de la demande allant au-delà de la simple commande.

Au final, cet ouvrage offre de quoi enrichir la boîte à outils des chercheurs, des intervenants et des professionnels en ressources humaines, non sans les avoir parfois quelque peu bousculés... 